\title{
TIME EVOLUTION OF THE LARGE-SCALE SOLAR MAGNETIC FIELD
}

\author{
MARTIN D. ALTSCHULER, GORDON NEWKIRK, JR., \\ and DOROTHY E. TROTTER \\ High Altitude Observatory, National Center for Atmospheric Research*, Boulder, Colo., U.S.A. \\ and \\ ROBERT HOWARD \\ Hale Observatories, Pasadena, Calif., U.S.A.
}

\begin{abstract}
The six years of data from the Mt. Wilson Magnetic Atlas were analyzed in terms of surface harmonics. Between 1959 and 1962 the dominant harmonic corresponded to a dipole lying in the plane of the equator ( 2 sectors). There was also a significant zonal harmonic in which both solar poles had the same magnetic polarity, opposite to that at the equator. From the end of 1962 through 1964, the harmonic corresponding to 4 sectors was dominant. In 1965 and 1966, the harmonic of the north-south dipole became significant.
\end{abstract}

The Mt. Wilson magnetic atlas (Howard et al., 1967) provides about six years of data of the line-of-sight component of the photospheric magnetic field. For any 28-day period (1 solar rotation), the data can be expanded in surface harmonics of the form $P_{n}^{m}(\theta) \cos m \phi$ and $P_{n}^{m}(\theta) \sin m \phi$. The coefficients of the Legendre series expansion are chosen by a least-mean-square fit to the given line-of-sight magnetic field data (Altschuler and Newkirk, 1969). We normalize the surface harmonics by the technique of Schmidt (1935) as in geomagnetic work (see Chapman and Bartels, 1940) because then the magnitude of the coefficient of a surface harmonic reflects the importance of that harmonic in representing the data. A normalization which makes the coefficient precisely proportional to the importance of the harmonic (in the sense that the average square value of the surface harmonic over the sphere is unity) (Schmidt, 1895) would give lower values for the coefficients of the large $n$ harmonics than would the Schmidt (1935) normalization. Thus the Schmidt (1935) normalization tends to overemphasize the importance of the harmonics of large $n$. We compared data samples using both the Schmidt (1935) and Schmidt (1895) normalization and found a rather small difference. A more detailed analysis will use a Schmidt (1895) normalization to evaluate the relative importance of the surface harmonics.

The geometrical interpretation of the spherical harmonics is simple. The harmonic $P_{n}^{m}(\theta) \cos m \phi$ (or $P_{n}^{m}(\theta) \sin m \phi$ ) is zero on $2 m$ different meridians equally spaced in longitude; a plot of the harmonic around a parallel of latitude will show $2 m$ zeroes. If $m \neq 0$, there are $2 m$ sectors, $m$ positive and $m$ negative. In the case $m=0$, the harmonic has a constant value along a parallel of latitude and the harmonic is called a zonal harmonic. The poles of the Sun can have a uniform magnetic polarity if and only if $m=0$. The integer $n-m$ is the number of times the surface harmonic goes

\footnotetext{
* The National Center for Atmospheric Research is supported by the National Science Foundation.
} 
to zero between (but not at) the poles. Along a meridian of longitude there are $n-m$ zeroes of the surface harmonic excluding those at the poles. Thus there are $n-m+1$ zones. If $n-m$ is an odd integer, the harmonic goes to zero at the equator, and the magnetic polarity changes when we cross the equator. If $n-m$ is an even integer, the region about the equator has a definite magnetic polarity. If $n-m=0$, there is a pure sector structure; that is, there are no zeroes along a meridian of longitude. The integer $n$ gives the index of the multipole; $n=0$ is a monopole, $n=1$ is a dipole, $n=2$ is a quadrupole, $n=3$ is an octopole, and $n=N$ is a ' $2^{N}$-pole'. In our procedure we assume there is no monopole component for the solar field, and the data is uniformly calibrated accordingly (Altschuler and Newkirk, 1969).

As an example, Figure 1 (top) shows the three surface harmonics $(n, m)=(7,0)$, $(n, m)=(7,5)$, and $(n, m)=(7,7)$. The first is a pure zonal harmonic $(m=0)$ with 7 zeroes along a meridian connecting the poles. Thus there are 8 zones. The second is a tesseral harmonic with $n-m+1=3$ zones and $2 m=10$ sectors, which divide the spherical surface into $2 m(n-m+1)=30$ separate surface regions of alternating

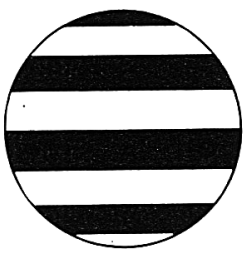

$\mathrm{P}_{7}^{\circ}(\theta)$

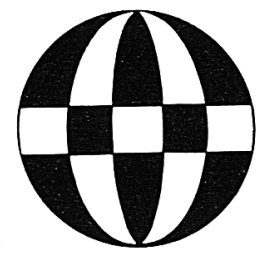

$P_{7}^{5} \cos 5 \phi$

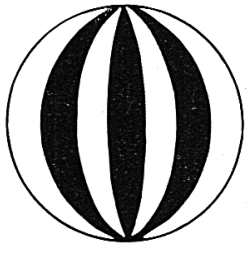

$P_{7}^{7} \cos 7 \phi$

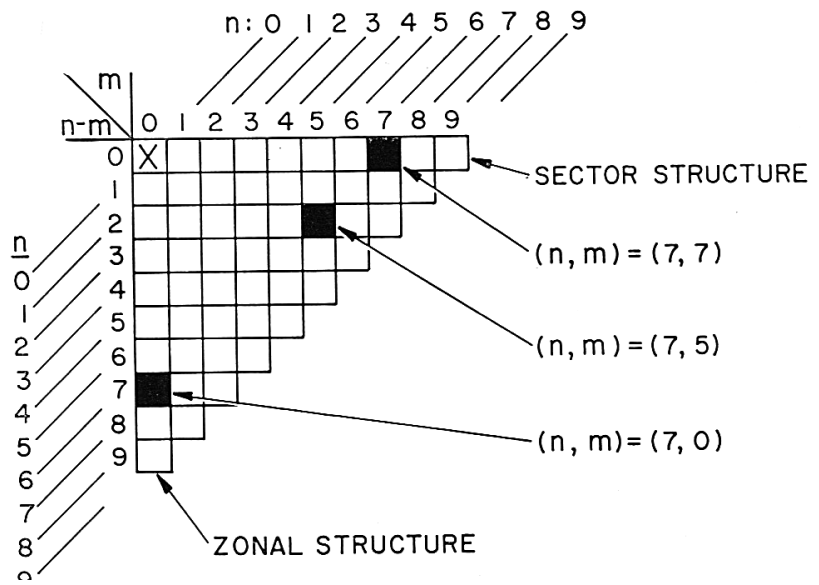

Fig. 1. Geometrical interpretation of surface harmonics. The integer $\boldsymbol{n}$ is the index of the multipole. The integer $m$ is half the number of (longitude) meridians on which the surface harmonic is zero. The integer $n-m$ is the number of (latitude) parallels between (but not including) the poles on which the surface harmonic is zero. If $n-m=0$, there is a pure sector structure. If $m=0$, there is a

pure zonal structure. 
magnetic polarity. The third is a pure sector structure harmonic $(n-m=0)$ with 14 zeroes along a parallel of latitude and 14 sectors (since $2 m=14$ ). Figure 1 (bottom) also shows the location of these harmonics on a graph of $n-m$ vs $m$. Since all 3 of these harmonics were chosen with $n=7$, they all lie along a diagonal of constant $n$.

To test the reliability of our procedure, we compared the harmonic analysis of the Mt. Wilson magnetic data with that of the Kitt Peak data for the same date 7 March 1970 (corresponding to an eclipse). The Kitt Peak magnetograph does not saturate even at sunspot field strengths whereas the Mt. Wilson magnetograph saturates at about $80 \mathrm{G}$. Although the Mt. Wilson data should be corrected for saturation, this was not done for the present comparison. Nevertheless, the agreement is remarkably good. Figure 2 (top) identifies the 5 largest harmonics (black squares) and their rank in magnitude (numbers in the squares). Actually, we omit the phase information and rank the magnitudes of $S_{n}^{m}=\left(g_{n}^{m}\right)^{2}+\left(h_{n}^{m}\right)^{2}$ where $g_{n}^{m}$ is the coefficient of $P_{n}^{m}(\theta) \cos m \phi$ and $h_{n}^{m}$ is the coefficient of $P_{n}^{m}(\theta) \sin m \phi$. The zonal and sector boundaries could be obtained by considering $g_{n}^{m}$ and $h_{n}^{m}$ separately; this has not yet been done.

Of the five largest harmonics in each data set, four agree in their $(n, m)$ identity. The other large harmonic had the same $n$ in both data sets but differed in the value of $m$ by $\Delta m=1$. Figure 2 (bottom) identifies the 11 largest harmonics (black and stippled squares). We find that 8 of the largest 11 harmonics have the same $(n, m)$ identity in both data sets. We conclude that the 5 largest surface harmonics are characteristic of the photospheric magnetic field and can be reliably obtained from existing magnetograph data.

The photospheric magnetic field for the eclipse date 7 March 1970 is interesting because there was no significant dipole component $(n=1)$. Both the north-south dipole component $(n=1, m=0)$ and the dipole component in the plane of the equator $(n=m=1)$ were relatively insignificant. There is a relatively weak quadrupole component $(n=2)$ and a strong octopole component $(n=3)$. The weakness of the dipole and quadrupole components means that the solar field decreased rather rapidly with radial distance on the eclipse date. The largest harmonic (which was twice as large as the next highest harmonic in the Schmidt (1935) normalization) corresponded to $n=m=8$, a very high order sector structure (16 sectors, 8 positive and 8 negative). There was no zonal structure (for which $m=0$ ). Consequently, neither pole of the Sun was characterized by a dominant magnetic polarity.

The six years of data from the Mt. Wilson magnetic atlas (Howard et al., 1967) were analyzed. Surface harmonic expansions were derived at equally spaced intervals (roughly every half rotation), except where gaps occurred in the data. Between 20 and 30 such harmonic expansions, spanning a time interval of about a year were considered as a single data period. We analyzed 6 data periods between 1959-1966. Figure 3 summarizes the harmonic structure for the 6 data periods. Each period is labelled with the last 3 digits of the first and last Carrington rotation and with the longitude at the center of the first and last data sample. For example, 417/170-431/0 means the period runs from (McMath) rotation 1417 centered at Carrington longitude $170^{\circ}$ to rotation 1431 centered at Carrington longitude $0^{\circ}$. The number of 'samples' is the 

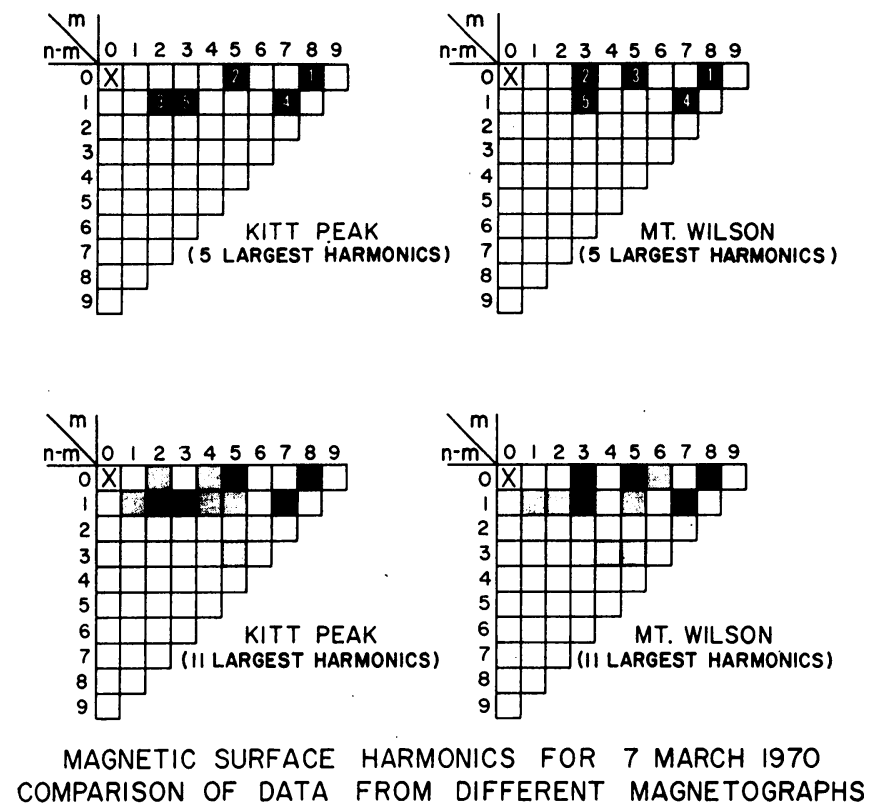

Fig. 2. Comparison of analysis using data from different magnetographs. The largest five surface harmonics are ranked in importance.
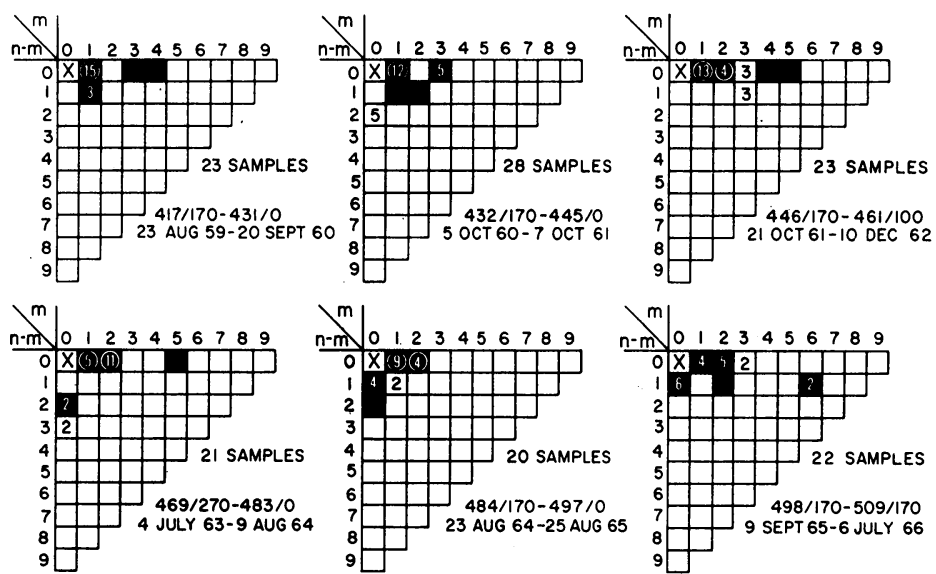

SOLID - 4 HARMONICS MOST OFTEN AMONG LARGEST 5

NUMBER - NUMBER OF TIMES HARMONIC WAS DOMINANT

CIRCLE - MORE THAN $2 \times$ NEXT HIGHEST HARMONIC

DOMINANT SURFACE HARMONICS FOR SOLAR MAGNETIC FIELD 1959-1966

Fig. 3. Roughly six years of dominant surface harmonics. Black boxes are harmonics which appear most often in top five in importance. Numbers in boxes give number of times harmonic was dominant.

Circle means harmonic was at least twice as large as the next largest harmonic on at least two occasions. 
number of harmonic expansions we computed for the data period. These samples are essentially uniformly spaced in time. In this analysis, the data were corrected for magnetograph saturation by a procedure (Altschuler and Newkirk, 1969) based on the ideas of Stenflo (1967). The harmonic shaded black are those which appear most
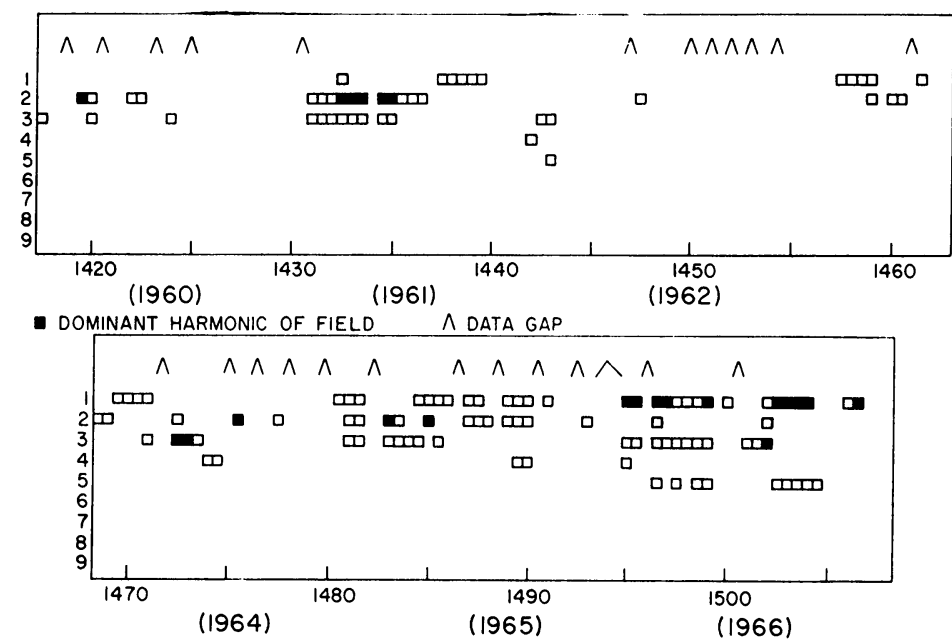

ZONAL STRUCTURE OF PHOTOSPHERIC MAGNETIC FIELD 1959-1966 ROTATIONS 1417 TO 1509

Fig. 4. Time variation of zonal harmonics. Plot of $n-m$ vs time. Blank box means harmonic was among the largest five harmonics. Black box means harmonic was the dominant harmonic.
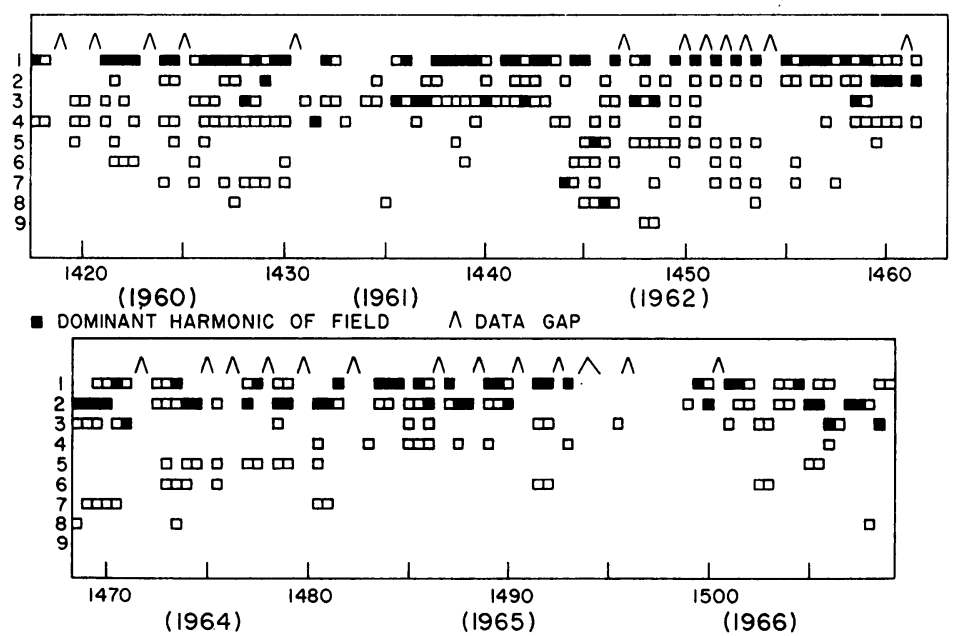

SECTOR STRUCTURE OF PHOTOSPHERIC MAGNETIC FIELD 1959-1966 ROTATION 1417 TO 1509

Fig. 5. Time variation of sector structure. Plot of $m$ vs time. (Same code as in Figure 4.) 
frequently among the largest 5 harmonics. The number in the box of a particular $(n, m)$ harmonic is the number of times the harmonic was the dominant (largest) harmonic of a data period. A circle around a number means that for at least two data samples in the data period the harmonic was at least twice as large as the next largest harmonic.

For most of the time between 1959 and 1962, the dominant harmonic was a dipole lying in the plane of the equator $(n=m=1)$. This harmonic corresponds to the simplest sector structure, with one hemisphere of positive polarity and the other hemisphere of negative polarity; the two sector boundaries are meridians of longitude $180^{\circ}$ apart. There was also a significant zonal harmonic $(n=2, m=0)$ in which both solar poles had the same magnetic polarity, opposite to that at the equator. At the end of 1962 and lasting through 1964 , the sector structure $(n=m=2)$ with 4 sectors ( 2 positive and 2 negative alternating in longitude) became the dominant harmonic largely because the dipole harmonic $(n=m=1)$ decreased in importance. The harmonic $(n=m=2)$ corresponds to the sector structure described by Wilcox and Ness (1965). With our technique we can establish that the sector structure of 1963 corresponded to $(n=m=2$, $n-m=0)$ and not to $(n=4, m=2, n-m=2)$. Both of these patterns are similar near the equator. In 1965 and 1966 in the post-minimum part of the solar cycle, the northsouth dipole component $(n=1, m=0)$ became significant. Figures 4 and 5 show the pure zonal and sector structures respectively as a function of time.

We point out that even during times of reliable data, there were rapid changes in the surface harmonic 'spectrum' of the photospheric magnetic field. Often significant changes in the dipole $(n=1)$ and quadrupole $(n=2)$ harmonics occurred in times of less than half a solar rotation. The low $n$ harmonics represent very large scale structures in the solar magnetic field. Rapid changes in such harmonics indicate the possible occurrence of very large and rapid changes in the energy of the solar magnetic field. Moreover, the coronal magnetic field at large distances would be most affected by the lowest $n$ harmonics. Therefore, any rapid changes in the low $n$ harmonics of the photospheric field would almost certainly affect the magnetic environment of the Earth.

In summary, we believe that our technique for examining all the magnetic surface harmonics should be useful in tracing the solar cycle.

\section{Acknowledgement}

The Atlas of Solar Magnetic Fields, which was our primary source of data, was funded in part by the Office of Naval Research (USA).

\section{References}

Altschuler, M. D. and Newkirk, Jr., G.: 1969, Solar Phys. 9, 131.

Chapman, S. and Bartels, Jr.: 1940, Geomagnetism, Clarendon Press, Oxford.

Howard, R., Bumba, V., and Smith, S. F.: 1967, Atlas of Solar Magnetic Fields 1959-1966, Carnegie Institute of Washington, Publication No. 626. 
Schmidt, A.: 1895, Abhandl. Bayer. Akad. Wiss. Munchen, II. Classe 19, 1.

Schmidt, A.: 1935, Tafeln der Normierten Kugelfunktionen, Sowie Formeln zur Entwicklung, Gotha, Engelhard-Reyer.

Stenflo, J. O.: 1967, Acta Univ. Lund. Sect. II, No. 35.

Wilcox, J. M. and Ness, N. F.: 1965, J. Geophys. Res. 70, 5793.

\section{Discussion}

Wilcox: The rapid change with time of the number of sectors found in your analysis is consistent with the observations of the interplanetary magnetic field showing that a new sector may be formed within a time interval of a few days. For example this might change the number of sectors from two to four. From our investigations it seems that the formation of a new sector may not be related to a flare.

Altschuler: Thank you.

Schatten: I think your analysis is very interesting. However, I would like to suggest that other orthonormal sets might more closely represent (and shed additional light) on the physical processes occurring in the photosphere. As Dr Newkirk showed, from the model of Leighton, there are backwards ' $C$ ' shaped structures on the Sun. This was observed by Dr Bumba and Dr Howard. This field would be represented by a series of Legendre polynomial terms. Perhaps it would be possible to combine the terms and thus form a new set that more closely resembles physical processes.

Altschuler: The Legendre polynomials are the natural orthonormal polynomials for a spherical surface. We can determine the sector and zonal structures, the magnetic polarity of the poles of the Sun, the multipole structure, and how the field decays with radial distance. Using the Legendre surface harmonic expansion, we have drawn many maps of the coronal field, and we feel confident that our method can delineate the photopheric boundaries between different magnetic polarities.

Ward: The axes of magnetic regions shift longitude as a function of latitude. Why did you not attempt to fit functions to the magnetic data which better represented this known feature of their appearance?

Altschuler: What we have tried to do is to summarize the photospheric magnetic field pattern in terms of a few parameters. The present Legendre polynomial procedure gives an excellent 'fit' to the available magnetic data and does it in an impartial way. Any other set of orthonormal polynomial functions should also work, but the geometrical interpretation might be more difficult.

Simon, M.: What is the ratio of the amplitude of the first five largest tesseral components, to the next five largest components? That is, thinking of your analysis as a power spectrum analysis in spherical harmonics, how rapidly does the 'power' decrease toward the higher spatial frequencies?

Altschuler: The most important surface hormonic (i.e. the surface harmonic with the largest coefficient) might have a value of $\mathbf{S}_{n}{ }^{m}$ about an order of magnitude greater than that of the sixth most important surface harmonic, and about two orders of magnitude greater than the eleventh ranking harmonic. The steepness of drop-off (i.e. the sharpness of the spectrum) is greatest during quiet years and is least during active years, probably because there is more randomness in the magnetic field during active years.

Vaiana: Do you have a map of the sectors for the March 7, 1970 eclipse?

Altschuler: We know the sectors and their phase relations but we have not made a sector map. We do have a map of the coronal magnetic field. 